\title{
Molecular quenching and relaxation in a plasmonic tunable system
}

\author{
Guillaume Baffou \\ Laboratoire de Photophysique Moléculaire (CNRS), Université Paris XI, F-91405 Orsay, France
}

Christian Girard and Erik Dujardin

Centre d'Elaboration des Matériaux et d'Etudes Structurales (CNRS), 29 Rue J. Marvig, F-31055 Toulouse, France

Gérard Colas des Francs

Institut Carnot de Bourgogne, UMR 5209 CNRS-Université de Bourgogne, 9 Avenue A. Savary, Boîte Postale 47870, F-21078 Dijon, France

\author{
Olivier J. F. Martin \\ Nanophotonics and Metrology Laboratory, Swiss Federal Institute of Technology Lausanne (EPFL), CH-1015 Lausanne, Switzerland
}

(Received 14 February 2008; published 19 March 2008)

\begin{abstract}
Molecular fluorescence decay is significantly modified when the emitting molecule is located near a plasmonic structure. When the lateral sizes of such structures are reduced to nanometer-scale cross sections, they can be used to accurately control and amplify the emission rate. In this Rapid Communication, we extend Green's dyadic method to quantitatively investigate both radiative and nonradiative decay channels experienced by a single fluorescent molecule confined in an adjustable dielectric-metal nanogap. The technique produces data in excellent agreement with current experimental work.
\end{abstract}

DOI: 10.1103/PhysRevB.77.121101

PACS number(s): 73.20.Mf, 34.35.+a, 07.79.Fc

The emission characteristic of a single quantum system excited by an external source of electrons or photons and interacting with metallic nanostructures is governed by a subtle balance between the radiative and nonradiative energy channels available in the vicinity of the metal. ${ }^{1}$ In many experiments, the excitation mechanism generally involves pure optical $^{2-9}$ or electro-optical ${ }^{10-12}$ processes. For example, in near field optics experiments, molecules deposited on dielectric surfaces are generally excited by an external laser beam or by the optical field generated at the tip apex of a near field optical microscope. ${ }^{4,6,7}$ In scanning tunneling microscope (STM), inelastic tunnel electronic events can also be used to excite fluorescent molecules. ${ }^{11,12}$ In spite of the fundamental difference between these two excitation modes, the fluorescence decay of the excited channels is governed by the same mechanisms. Actually, three emission regimes can be defined by comparing the absolute value of the tip-molecule distance to the fluorescence wavelength $\lambda_{0}$. (i) In the wave zone, ${ }^{1}$ emission rate smoothly oscillates around the free space value $\Gamma_{0}$ due to interferences with the sample surface. (ii) When entering the subwavelength distance range, the total decay rate of the molecule is significantly modified (enhanced or partially canceled) by its transition dipole image. ${ }^{13}$ (iii) At even shorter distances, ranging from few nanometers to the physical contact with the metallic structure, a second mechanism tends to increase the total decay rate. This effect, which is responsible for fluorescence quenching, corresponds to the opening of a new decay channel by absorption of fluorescence photons in the metallic structure itself. $6,7,14,15$ Over the past few years, these well-known physical concepts have been applied to possible new plasmonic devices, with the aim of achieving (i) high density integrated photonic components, (ii) low threshold detection of biological and chemical species, and to supply (iii) new molecular-scale characterization techniques for biomolecules. ${ }^{16}$ In this rapidly growing field, systems consisting of isolated molecules interacting with noble metal nanostructures are used as prototype models to test and validate new schemes for active plasmonic devices. Recently, a new step toward molecular plasmonics was achieved with the demonstration of the control of fluorescent resonant energy transfer through thin layers of plasmonic materials. ${ }^{17}$ These experiments use the excitation of surface plasmons to enhance and control the efficiency of the optical transfer between two remote molecular sites. Such controlled light transfer in highly reduced geometries can pave the way for future optical technologies, integrable and scalable down to molecular dimensions. ${ }^{18}$ Finally, in a closely related context involving the spatial control of sharp metal tips or the effective realization of nanoparticle plasmon waveguides, single molecules are often used as efficient local sensors for the plasmonic near-field intensity. ${ }^{19}$ In this Rapid Communication, we present a fully self-consistent framework relevant for the interpretation of recent experiments involving molecular quenching and relaxation in a plasmonic tunable nanogap. Our model includes the complex physics involved in quantum systems coupled with plasmonic environments. In the presence of plasmonic devices that sustain localized plasmon modes, such an analysis requires a specific attention to properly include local field enhancements, Ohmic losses, and radiative decay rates. In particular, we apply Green's dyadic method (also called field-susceptibility technique) to treat these different phenomena in the presence of finite plasmonic structures.

In near-field experimental setups based either on electrons or photons detection, the central element is the tip of a metallic stylus that is raster scanned across the sample. We consider the case of such a sharp metallic probe facing a plane dielectric surface on which $N$ identical fluorescing molecules have been adsorbed (Fig. 1). The tip is modeled as a paraboloid enclosed in a surface of equation $A x^{2}+A y^{2}=z$. The pa- 


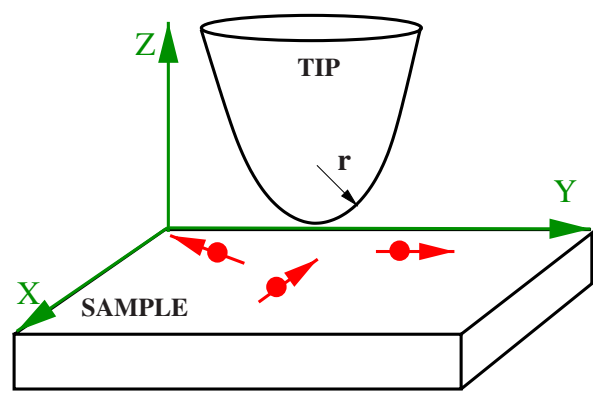

FIG. 1. (Color online) Schematics of the geometry used to model light emission by molecules located in the gap between a paraboloid metallic tip and a planar dielectric surface. The curvature radius $\mathbf{r}$ of the tip apex is fixed at $8 \mathrm{~nm}$.

rameter $A$ can then be adjusted to modify the sharpness of the tip. In the numerical scheme used in this Rapid Communication, the physical volume limited by this surface will be discretized on a Cartesian grid stacking layers of meshes in a closed packed arrangement. When we neglect any optical coupling between the individual molecules, the total fluorescence energy released by the sample at the frequency $\omega_{0}$ is given by

$$
I\left(\mathbf{R}_{\text {tip }}\right)=\sum_{i=1}^{N} \hbar \omega_{0} \rho\left(\mathbf{r}_{i}, \mathbf{u}_{i}, \omega_{0}\right) \Gamma\left(\mathbf{r}_{i}, \mathbf{u}_{i}, \omega_{0}\right),
$$

where $\mathbf{R}_{\text {tip }}$ labels the position of the tip apex, $\mathbf{r}_{i}$ represents the location of the $i$ th molecule deposited on the surface, and the units vectors $\mathbf{u}_{i}$ have been introduced to define the orientations of their transition dipole moment $\mathbf{P}_{e g}$ with respect to an absolute surface frame. In addition, the two factors $\rho\left(\mathbf{r}_{i}, \omega_{0}\right)$ and $\Gamma\left(\mathbf{r}_{i}, \omega_{0}\right)$ define the population rate and the total decay rate associated with the $i$ th molecule, respectively. Both quantities depend on the orientation and the location of the molecules. The first one, $\Gamma\left(\mathbf{r}_{i}, \mathbf{u}_{i}, \omega_{0}\right)$, is given by

$$
\Gamma\left(\mathbf{r}_{i}, \mathbf{u}_{i}, \omega_{0}\right)=\Gamma_{0}+\frac{2 P_{e g}^{2}}{\hbar} \operatorname{Im}\left\{\mathcal{S}\left(\mathbf{r}_{i}, \mathbf{r}_{i}, \omega_{0}\right)\right\}: \mathbf{u}_{i} \mathbf{u}_{i} .
$$

The field-susceptibility $\mathcal{S}\left(\mathbf{r}_{i}, \mathbf{r}_{i}, \omega_{0}\right)$ that enters the total decay rate is defined in Ref. 20. This dyadic tensor describes the dipolar response of the whole tip-surface system. It obeys Dyson's equation that can be numerically solved with an algorithm that relies on a three-dimensional discretization of the metal tip. ${ }^{21}$

In Eq. (1), the population rate $\rho\left(\mathbf{r}_{i}, \mathbf{u}_{i}, \omega_{0}\right)$ is directly related to the mechanism we used to excite the molecules. For example, in the case of optical excitations with a laser light source at the frequency $\omega_{L}$, it can be easily obtained from the Maxwell-Bloch equation scheme (see Ref. 20). In particular, a two-level molecular model predicts the following Lorentzian law:

$$
\rho\left(\mathbf{r}_{i}, \mathbf{u}_{i}\right)=\frac{\Omega^{2}\left(\mathbf{r}_{i}, \mathbf{u}_{i}, \omega_{L}\right)}{4 \delta_{L}^{2}+\Gamma^{2}\left(\mathbf{r}_{i}, \mathbf{u}_{i}, \omega_{0}\right)+2 \Omega^{2}\left(\mathbf{r}_{i}, \mathbf{u}_{i}, \omega_{L}\right)},
$$

where $\delta_{L}=\omega_{L}-\omega_{0}$ is the detuning factor and $\Omega\left(\mathbf{r}_{i}, \mathbf{u}_{i}, \omega_{0}\right)$ defines the Rabi frequencies at the molecule locations $\mathbf{r}_{i}$. As indicated by Eq. (3), the molecular population rate $\rho$ intricately depends both on the Rabi frequency and the excited state decay rate. The Rabi frequency is proportional to the optical near-field intensity at the molecule location. The saturation regime occurs when this quantity is significantly larger than $\Gamma$. In the case of a two-level model, the population rate stabilizes around the ratio of $1 / 2$ for zero detuning. In the following numerical applications, we will assume that this saturation regime has been reached.

When the tip enters the subwavelength approach distance range, the total decay rate $\Gamma\left(\mathbf{r}_{i}, \mathbf{u}_{i}, \omega_{0}\right)$ of the molecules is significantly enhanced compared to its free-space value $\Gamma_{0}$. For a selected molecule, which is located at the position $\mathbf{r}_{i}$, the enhancement factor can be derived from

$$
\eta\left(\mathbf{r}_{i}, \mathbf{u}_{i}, \omega_{0}\right)=\Gamma\left(\mathbf{r}_{i}, \mathbf{u}_{i}, \omega_{0}\right) / \Gamma_{0}=1+\frac{2 P_{e g}^{2}}{\hbar \Gamma_{0}} \operatorname{Im}\left\{\mathcal{S}\left(\mathbf{r}_{i}, \mathbf{r}_{i}, \omega_{0}\right)\right\}: \mathbf{u}_{i} \mathbf{u}_{i} .
$$

This well-known phenomenon is associated with an increase (or decrease) of the local density of available photonic states when the tip is approaching the molecule. ${ }^{4}$ However, in the vicinity of dissipative materials, a second mechanism will significantly reduce the radiative decay rate. This effect, responsible for fluorescence quenching, corresponds to the absorption of fluorescence photons by the metallic structure itself. To compute this dissipated power $Q\left(\mathbf{R}_{t i p}\right)$, we consider that each emitting molecule behaves as a fluctuating dipole defined by

$$
\mathbf{m}_{i}(t)=P_{e g} \mathbf{u}_{i} \cos \left(\omega_{0} t\right) .
$$

In turn, these dipoles give rise to an electric field inside the metal,

$$
\mathbf{E}_{t i p}\left(\mathbf{r}, \omega_{0}\right)=\sum_{i} \mathcal{S}\left(\mathbf{r}, \mathbf{r}_{i}, \omega_{0}\right) \cdot P_{e g} \mathbf{u}_{i},
$$

which oscillates at the fluorescence frequency $\omega_{0}$. The dissipated power $Q\left(\mathbf{R}_{t i p}\right)$ is given by the integral of the square modulus of $\mathbf{E}_{t i p}\left(\mathbf{r}, \omega_{0}\right)$ over the volume occupied by the tip. This scalar quantity can be normalized by the free-space power $\hbar \omega_{0} \Gamma_{0}$. After using Eq. (6), it reads

$$
\overline{\mathcal{Q}}\left(\mathbf{R}_{t i p}\right)=\frac{3}{2 k_{0}^{3}} \int_{t i p} \sum_{i} \operatorname{Im}\left[\chi\left(\omega_{0}\right)\right]\left|\mathcal{S}\left(\mathbf{r}, \mathbf{r}_{i}, \omega_{0}\right) \cdot \mathbf{u}_{i}\right|^{2} d \mathbf{r},
$$

where $\operatorname{Im}\left[\chi\left(\omega_{0}\right)\right]$ represents the imaginary part of the metal susceptibility. In the numerical work presented in this Rapid Communication, we have chosen a paraboloid-shaped gold tip facing a planar glass surface (optical index $n=1.5$ ) located at the coordinate $Z=0$. The tip displays a circular cross section in the $(X O Y)$ plane. The aperture angle and tip radius of the paraboloid are fixed at $30^{\circ}$ and $8 \mathrm{~nm}$, respectively. A volume discretization of the tip based on a hexagonal mesh is used to compute the retarded field susceptibility around the system. The molecules, which are characterized by a fluorescence wavelength $\lambda_{0}$ and a natural linewidth $\Gamma_{0}$, are adsorbed at $1 \mathrm{~nm}$ from the glass substrate. From Eqs. (1), (4), and (7), we can deduce the total fluorescence signal collected by a remote detector placed in the far-field zone above the sample, ${ }^{22}$ 


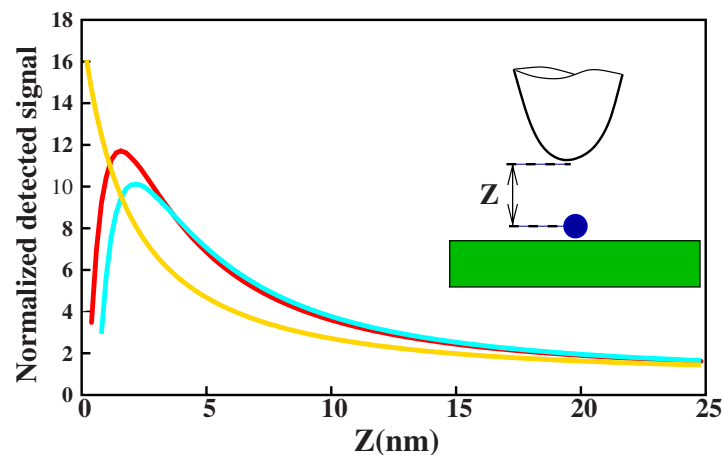

FIG. 2. (Color online) Total fluorescence signal computed as a function of the tip-sample distance. Three different fluorescence wavelengths are investigated: red curve (dark gray) $\lambda_{0}=620 \mathrm{~nm}$, cyan curve (gray) $\lambda_{0}=534 \mathrm{~nm}$, and yellow curve (light gray) $\lambda_{0}$ $=780 \mathrm{~nm}$. The molecule is perpendicular to the surface.

$$
I_{d e t}\left(\mathbf{R}_{t i p}\right)=\hbar \omega_{0} \Gamma_{0} \sum_{i=1}^{N} \rho\left(\mathbf{r}_{i}, \mathbf{u}_{i}, \omega_{0}\right)\left[\eta\left(\mathbf{r}_{i}, \mathbf{u}_{i}, \omega_{0}\right)-\overline{\mathcal{Q}}\left(\mathbf{R}_{t i p}\right)\right] .
$$

This expression [Eq. (8)] together with Eq. (7) has been implemented numerically on a parallel supercomputer to investigate several photophysical properties of molecules coupled with plasmonic tips. In Eqs. (8) and (7), the field susceptibility $\mathcal{S}$ is computed outside and inside the metal by iteratively solving a sequence of Dyson's equations. The corresponding numerical results are shown in Figs. 2-5. In Fig. 2 , a series of three typical tip-molecule distance curves computed for three different fluorescence wavelengths $\lambda_{0}$ shows the dramatic variation of the far-field intensity [Eq. (8)] when the molecule approaches the metal quenching zone.

We observe that significant fluorescence quenching occurs at very short distances from the metallic tip [e.g., few nanometers for the red (dark gray) and cyan (gray) curves in Fig. 2]. In these two examples, the dissipated power totally cancels the radiative emission rate before reaching the physical contact of the molecule with the metal. Conversely, at $\lambda_{0}=780 \mathrm{~nm}$, this behavior does not occur because the imagi-

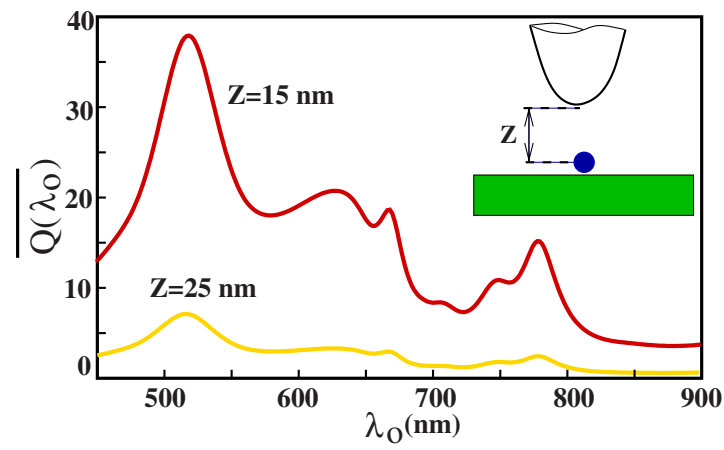

FIG. 3. (Color online) Variation of the power dissipated by an arbitrary molecule versus its fluorescence wavelength $\lambda_{0}$. The power is normalized to the power $\hbar \omega_{0} \Gamma_{0}$ delivered by the molecule in vacuum.

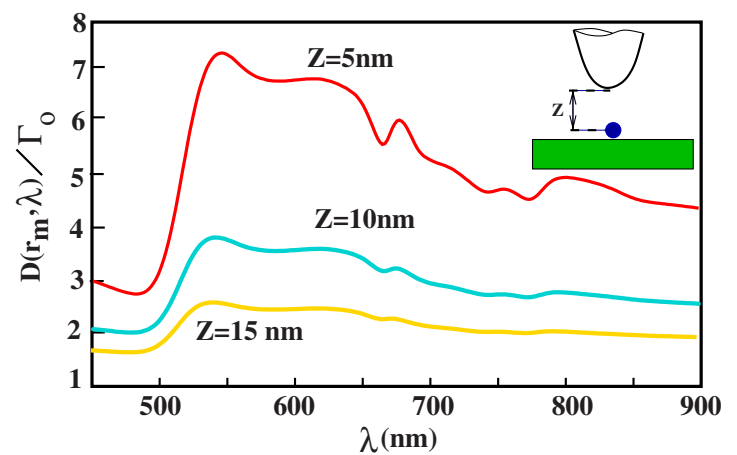

FIG. 4. (Color online) Evolution of the far-field intensity as a function of the fluorescence wavelength $\left(\lambda_{0}\right)$. Three approach distances $Z$ are considered: red curve (dark gray) $Z=5 \mathrm{~nm}$, cyan curve (gray) $Z=10 \mathrm{~nm}$, and yellow curve (light gray) $Z=15 \mathrm{~nm}$. The signal is normalized to the power $\hbar \omega_{0} \Gamma_{0}$ delivered by the molecule in vacuum.

nary part of the gold dielectric constant decreases in this frequency range.

Very recently, Issa and Guckenberger ${ }^{23}$ have analyzed the role of the surface plasmon polaritons mode in the relaxation processes. They separate the local energy transfer to the very tip and the long range energy losses during the propagation of the plasmon surface wave along the tip-air interface. In the present analysis, these two contributions are taken into account in the nonradiative rate [Eq. (7)]. Generally, let us note that because of the plasmon propagation at long distance, direct calculation of the radiative decay rate is extremely difficult since it includes the energy transported by the plasmon mode that should be totally dissipated by Joule effect for infinitely extended gold tip. ${ }^{23,24}$ More generally, when exploring the visible range spectrum, the simulations indicate that the fluorescence enhancement varies between 10 and 16 . We conclude that energy losses, which are intrin-

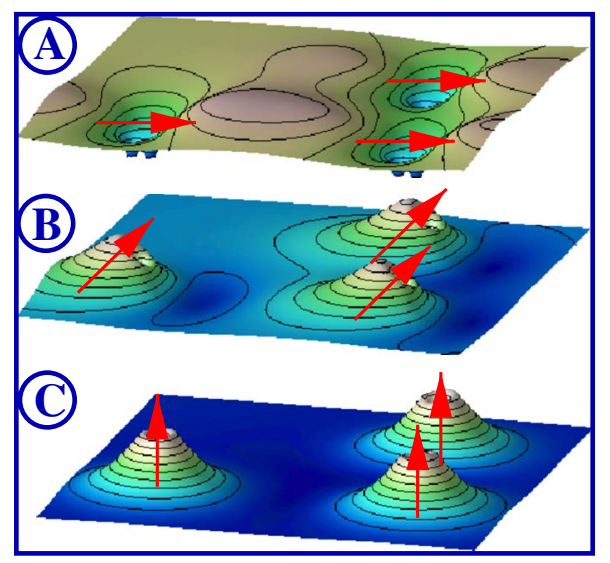

FIG. 5. (Color online) Sequence of three maps of the fluorescence intensity scattered in the far-field zone The rainbow color (gray) scale increases from blue (dark gray) to pale red (light gray). The tip-molecule distance is fixed at $2.5 \mathrm{~nm}$ and three orientations of the transition dipole moments (red arrows) are investigated: (A) parallel to the sample surface, (B) tilted with an angle of $45^{\circ}$, and (C) perpendicular to the surface. 
sic to the metal tip, considerably reduce the expected fluorescence amplification effect. The amplitude variation of these losses can be predicted with our model. In the visible frequency range, two spectra of the power dissipated in the gold tip by a single molecule are plotted in Fig. 3 as a function of the fluorescence wavelength $\lambda_{0}$. In these two examples, the molecule is perpendicularly aligned to the surface. We observe well-contrasted signatures of the power dissipated in the metal as a function of the fluorescence wavelength $\lambda_{0}$. Apart from the main plasmon resonance of the tip $(\simeq 520 \mathrm{~nm})$, the number of fluorescence photons absorbed by the metal increases. This phenomenon produces an enhancement of the normalized factor $\overline{\mathcal{Q}}$ between 8 and 38 when the tip-molecule distance decreases from 25 to $15 \mathrm{~nm}$. This results can be compared with the spectra of the total signal collected by a remote sensor (see Fig. 4). Figure 4 shows that the spectral variation of this signal-described as the difference between two quantities of the same magnitude order $\left[I\left(\mathbf{R}_{t i p}\right)\right.$ and $\left.Q\left(\mathbf{R}_{t i p}\right)\right]$-is generally smoother than the variation of the sole dissipated power $Q\left(\mathbf{R}_{\text {tip }}\right)$. With the gold tip considered here, the optimal enhancement effect is obtained when working with fluorescence wavelengths $\lambda_{0}$ between 520 and $630 \mathrm{~nm}$.

To conclude this analysis, Fig. 5 shows a sequence of three maps for the normalized detected signal. The maps are computed by scanning the tip in the constant distance mode. The planar glass sample supports three identical fluorescing molecules for which three molecular orientations have been investigated, keeping the same tip-sample distance $(2.5 \mathrm{~nm})$. In the first map [Fig. 5(a)], the transition dipole moments are parallel to the surface. In this geometry, individual molecules appear as elongated depressions perpendicularly oriented to their transition dipoles and surrounded by two-lobed patterns. Furthermore, the two closest molecules simulated in this figure exhibit a pattern with three lobes and two depressions, which is in fairly good agreement with the results of Ref. 14. In the second map Fig. 5(b), the dipole moments are tilted out of the sample plane with an angle of $45^{\circ}$. Here, each molecule produces an asymmetrical protrusion with a small depression localized on its right side. Finally, these patterns gradually transform into regular truncated conical protrusions with a small depression on their apex when the molecules are perpendicularly aligned to the surface. These orientational behaviors are found in perfect agreement with recent experimental data measured in similar tip-sample geometries. $6,7,14$

To summarize, we have described a versatile scheme well suited for describing and predicting photophysical mechanisms triggered by the vicinity of complex plasmonic devices. When applied to an adjustable plasmonic nanogap geometry, this framework successfully reproduces experimental signals. Consequently, this numerical tool can be used to support current experimental work developed in the field of molecular plasmonics. In particular, it can be applied to any geometrical configuration, to the accurate computation of the dissipation losses, and to the research of strategies to minimize them. Finally, by introducing an appropriate model for the excitation rate parameter $\rho\left(\mathbf{r}_{i}, \mathbf{u}_{i}\right)$, the method could be extended to the description of optical relaxation of single molecules located in an STM nanogap. ${ }^{10,11}$

This work was supported in part by the European Network of Excellence (NoE) Plasmo-Nano-Devices (Contract No. 507879).
${ }^{1}$ R. R. Chance, A. Prock, and R. Silbey, Adv. Chem. Phys. 37, 1 (1978).

${ }^{2}$ F. D. Stefani, K. Vasilev, N. Bocchio, N. Stoyanova, and M. Kreiter, Phys. Rev. Lett. 94, 023005 (2005).

${ }^{3}$ B. C. Buchler, T. Kalkbrenner, C. Hettich, and V. Sandoghdar, Phys. Rev. Lett. 95, 063003 (2005).

${ }^{4}$ W. Trabesinger, A. Kramer, M. Kreiter, B. Hecht, and U. P. Wild, Appl. Phys. Lett. 81, 2118 (2002).

${ }^{5}$ A. Cvitkovic, N. Ocelic, J. Aizpurua, R. Guckenberger, and R. Hillenbrand, Phys. Rev. Lett. 97, 060801 (2006).

${ }^{6}$ P. Anger, P. Bharadwaj, and L. Novotny, Phys. Rev. Lett. 96, 113002 (2006).

${ }^{7}$ S. Kuhn, U. Hakanson, L. Rogobete, and V. Sandoghdar, Phys. Rev. Lett. 97, 017402 (2006).

${ }^{8}$ G. P. Wiederrecht, J. E. Hall, and A. Bouhelier, Phys. Rev. Lett. 98, 083001 (2007).

${ }^{9}$ O. Labeau, Ph. Tamarat, H. Courtois, G. S. Agarwal, and B. Lounis, Phys. Rev. Lett. 98, 143003 (2007).

${ }^{10}$ J. Aizpurua, G. Hoffmann, S. P. Apell, and R. Berndt, Phys. Rev. Lett. 89, 156803 (2002).

${ }^{11}$ M. Sakurai, C. Thirstrup, and M. Aono, Phys. Rev. Lett. 93, 046102 (2004).

${ }^{12}$ X. H. Qiu, G. V. Nazin, and W. Ho, Phys. Rev. Lett. 93, 196806 (2004)
${ }^{13}$ W. Barnes, J. Mod. Opt. 45, 661 (1998).

${ }^{14}$ H. G. Frey, S. Witt, K. Felderer, and R. Guckenberger, Phys. Rev. Lett. 93, 200801 (2004).

${ }^{15}$ G. Colas des Francs, C. Girard, M. Juan, and A. Dereux, J. Chem. Phys. 123, 174709 (2005).

${ }^{16}$ T. Neumann, M.-L. Johansson, D. Kambhampati, and W. Knoll, Adv. Funct. Mater. 12, 575 (2002); G. Stengel and W. Knoll, Nucleic Acids Res. 33, 7 (2005); J. R. Lakowicz, Anal. Biochem. 337, 171 (2005).

${ }^{17}$ P. Andrews and W. L. Barnes, Science 306, 1002 (2004).

${ }^{18}$ D. E. Chang, A. S. Sörensen, P. R. Hemmer, and M. D. Lukin, Phys. Rev. Lett. 97, 053002 (2006).

${ }^{19}$ H. Ditlbacher, J. R. Krenn, N. Felidj, B. Lamprecht, G. Schider, M. Salerno, A. Leitner, and F. R. Aussenegg, Appl. Phys. Lett. 80, 404 (2002).

${ }^{20}$ C. Girard, O. J. F. Martin, G. Leveque, G. Colas des Francs, and A. Dereux, Chem. Phys. Lett. 404, 44 (2005).

${ }^{21}$ O. J. F. Martin and M. Paulus, J. Microsc. 205, 147 (2002).

${ }^{22}$ For simplicity, we have omitted the angular dependency of the detected signal.

${ }^{23}$ During the review process, a related article has been published: N. Issa and R. Guckenberger, Opt. Express 15, 12131 (2007).

${ }^{24}$ F. Kaminski, V. Sandoghdar, and M. Agio, Chem. Abstr. 4, 635 (2007). 